\title{
Temporary Bronchial Occlusion in Fistulous Forms of Bacterial Lung Destruction in Children
}

\author{
Chuliev MS, Uglonov IM, Narbaev TT, Xotamov XN, Pulatov FT, Barotov FT and Nasirov MM* \\ Tashkent Pediatric Medical Institute, Uzbekistan
}

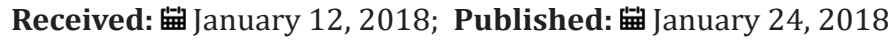

*Corresponding author: Mansur Nasirov, Tashkent Pediatric Medical Institute, Uzbekistan

\begin{abstract}
Despite the improvement of methods for diagnosis and treatment of bacterial lung destruction (BDL) in children, there are many cases of complicated fistula development. Bronchopleural fistulas in children mostly develops due to breakthrough into the pleural cavity of the lung abscesses communicating with the bronchus [1,2]. One of the main factors conditioning pulmonary collapse and its non-expansion, even with drainage of the pleural cavity, is the functioning of peripheral Bronchopleural fistulas (BPF) and the absence of a bronchial system due to this tightness. Principles of treatment of patients with Bronchopleural fistulas derive from an understanding of the cause of fistula development, the mechanisms of development of respiratory failure and disruption of homeostasis. The main reasons for the development of respiratory failure in Bronchopleural fistulas is the development of the lung leakage syndrome, which in turn inhibits the spreading of the lung [3,4].
\end{abstract}

Methods of surgical treatment of pleural empyema aimed at evacuation of purulent contents from the pleural cavity and foci of lung destruction can be divided into 2 types: "open" - with the use of thoracotomy and "closure" or methods of minimally invasive surgery [5-7]. The latter include temporary bronchial occlusion under the video-control (VATS). Temporary bronchial occlusion is an artificial disconnection of the pathological Bronchopleural communication that occurs when purulent lung destruction is complicated by pyopneumothorax.

Treatment in conditions of the remaining fistula becomes very long, often ineffective and often ends in a large and traumatic operation. The first successful endobronchial occlusion was applied by a Polish specialist. Rafinski (1965), who is rightly considered a pioneer in this field [8]. A modified method of occlusion of the bronchus proposed by Russian specialist V.I. Geraskin (1974) gave the possibility of bronchoscopic treatment of peripheral Bronchopleural fistulas. Studies carried out on 12 patients showed that after occlusion it was possible to restore the tightness of the bronchial tree and spread the healthy parts of the lungs [9].

Thus, the detection of the bronchus with pleural fistula in children remains a complicated and unsolved problem, which requires further searching for simpler, more reliable and safe methods [10-12]. According to the references, the method of searching fistula before occlusion and temporary bronchial occlusion give us the possibility of not only eliminating the flow of air into the pleural cavity, but also preventing aspiration of purulent contents in the healthy parts of the lungs. This creates conditions for expanding the collapsed lung, healing bronchial fistulas, normalizing pulmonary ventilation and gas composition of the blood.

\section{Purpose}

To conduct a clinical, radiological and bronchological evaluation of the effectiveness of temporary bronchial occlusion in the treatment of Bronchopleural fistula in children in the near and long-term periods.

\section{Material and Methods}

147 patients with bacterial destruction of the lungs (BDL) were managed from 2006 to 2016 in the Tashkent Pediatric Medical 
Institute's clinic. In $32(22 \%)$ of them at the age from 7 to 16 years, various variants of temporary occlusion have been used (due to the development of Bronchopleural fistula) - occlusion of lobar or segmental bronchi. Treatment of BDL was carried out according to the accepted protocol, which includes drainage of the pleural cavity (chest tube). In 21 (65\%) cases, the right lung was affected, in 11 (35\%) left. During the period of the research, a bilateral lesion was not observed. In $8(25 \%)$ patients the changes were localized in the upper lobe, 19 (59\%) in the lower lobe and 5 (16\%) showed polysegmentary lung destruction.

All patients, in addition to routine diagnostic methods, received chest radiographs in the direct and lateral projection, bronchoscopy before and after the operation, including sanative bronchoscopy prior to surgery. Multislice computed tomography was also performed. In the postoperative period, spirometry was performed to determine the functioning of lung tissue, as well as Echo-CG to determine blood flow in a pulmonary circulation.

Bronchoscopy was followed by bronchial lavage of affected sites. In all cases, temporary occlusion was performed after drainage of the pleural cavity. In this case, we preferred to give rigid bronchoscopy with general anesthesia with the introduction of an aseptic occludor (large porous foam impregnated with povidoneiodine and tantalum) in the fistula bronchus. The bronchoscopic temporary bronchial occlusion was performed under the thoracoscopic control, to examine the pleural cavity and identify peripheral bronchial fistulas. Thoracoscopy was performed under intratracheal anesthesia simultaneously with bronchoscopy. Drainage of the pleural cavity was preserved after the operation.

Occludor was in the lumen of the bronchial tree on average from 7 to 16 days (an average of 11). Indication for the removal of the occludor was the spreading of the lung, the elimination of pneumothorax. The diameter of the occludor was selected in accordance with the fistulous course, established bronchoscopically. Indications for the removal of the occludor were: normalization of spirometry and pulmonary blood flow, improvement of ventilation. For the analysis of long-term results, a chest X-ray in a direct projection and bronchography were performed.

\section{Results}

The clinical effect of the temporary bronchial occlusion was formed by a certain pattern of changes. For example, in the first days there was a primary reaction to occlusion. Thus, in $12(37.5 \%)$ patients, normalization of the function of external respiration was noted in the first 24 hours (RR decreasing, the lungs ventilation volume increased, and the tidal volume decreased to $25 \%$ comparing to normal). In 19 (59\%) patients, the primary reaction phase lasted up to 2 days. It was characterized by the same clinical manifestations. In 1 (3.5\%) patient, the initial adaptation to occlusion lasted for 4 days. Over the next 10 days, 18 (56\%) experienced a gradual improvement in general condition, stabilization of the basic parameters of respiration and circulation, which was accompanied by an increase in the use of oxygen, an increase in its consumption by $30-35 \%$, normalization of PaCO2 and $\mathrm{PaO} 2$, and stabilization of the general condition and depression of the inflammatory processes in the lung. In 13 (40.5\%) patients described clinical manifestations developed on day 14 , in 1 (3.5\%) patient on day 21 . All patients $(100 \%)$ had normal body temperature in the first 3 days, dyspnea was decreased, purulent discharge from the pleural cavity ceased.

On the x-ray control, in $28(87.5 \%)$ patients, on the 2nd day, uncontrolled areas of the lung and the inclusion of them into the respiratory function were noted. There was a reduction and a significant decrease in pneumothorax. In $3(9 \%)$ patients, the spread occurred on day 3 and in $1(3.5 \%)$ patient by day 5 , which was associated with late admission of the patient and the presence of intercurrent diseases. When the children were examined at distant times (from 6 to 12 months), all patients showed persistent pulmonary spreading, normalization of the vascular pattern, displacement of the mediastinum to the healthy side, reduction of the intercostal spaces and lifting of the diaphragm.

When bronchography, conducted a year later, all patients had normal bronchial pattern without signs of a stitch of contrast in the pleural cavity. On US examination of the pulmonary system, 26 (82\%) showed a significant decrease in anatomic shunting of blood in the small circulation (more than 3 times), in 3 (9\%) patients there was a decrease in pulmonary artery pressure and in 3 (9\%) of patients normalization of pressure in the pulmonary artery. According to Echo-CG, in $21(66 \%)$ the shock volume of the heart increased to $60-70 \mathrm{ml}$, the minute volume of the heart increased to 7-8 l/min.

Based on the material available, there are 2 clinical cases below.

Case 1: Patient T. 7 years old, boy. He admitted with complaints of cough, shortness of breath, fever of up to $40 \mathrm{0C}$. He underwent of draining of the pleural cavity (before admission) from which the air-leak was noted. The disease began 2 months before admission. The bacterial destruction of the lungs, pyopneumothorax, and Bronchopleural fistula was diagnosed in clinic on a residence. On the $\mathrm{x}$-ray, free gas and the presence of fluids in the pleural cavity was noted.

According to the indications, CT-scanning was performed (Figures 1-3). Bacterial destruction of the lung was confirmed. Pulmonary-pleural form. Pyopneumothorax and right-sided empyema. When all the diagnostic procedures were carried out, bronchoscopy, lavage of the bronchial tree, occlusion of the right upper lobe bronchus was performed. After that, the separation of air from the drainage pipe immediately stopped. The drainage tube functioned for 8 days. After 12 days, bronchoscopy, removal of the occludor, lavage of the bronchial tree was performed. On $\mathrm{x}$-ray control (Figure 4) complete expansion of the lungs, absence of Bronchopleural fistula was noted.

Case 2: Patient B., 3 month of age. Admitted with complaints of cough, shortness of breath (RR over 70), fever (up to $40 \mathrm{OC}$ ), and weakness. Patient experienced these complaints for 2 month. General condition of the patients was severe as described previously. 
Additional muscles took part in breathing, crackles were noted in auscultation. On CT (Figure 5) bilateral bronchopneumonia, rightsided pneumothorax Bronchopleural fistula. After preoperative management and stabilizing patient's condition, right-sided thoracocentesis was performed. After 10 days bronchoscopy, bronchial lavage and temporary bronchial occlusion was done. Occludor was removed after 10 days. On x-ray control: absence of free air in pleural cavity. Complete expansion of the pleural cavity (Figure 6)

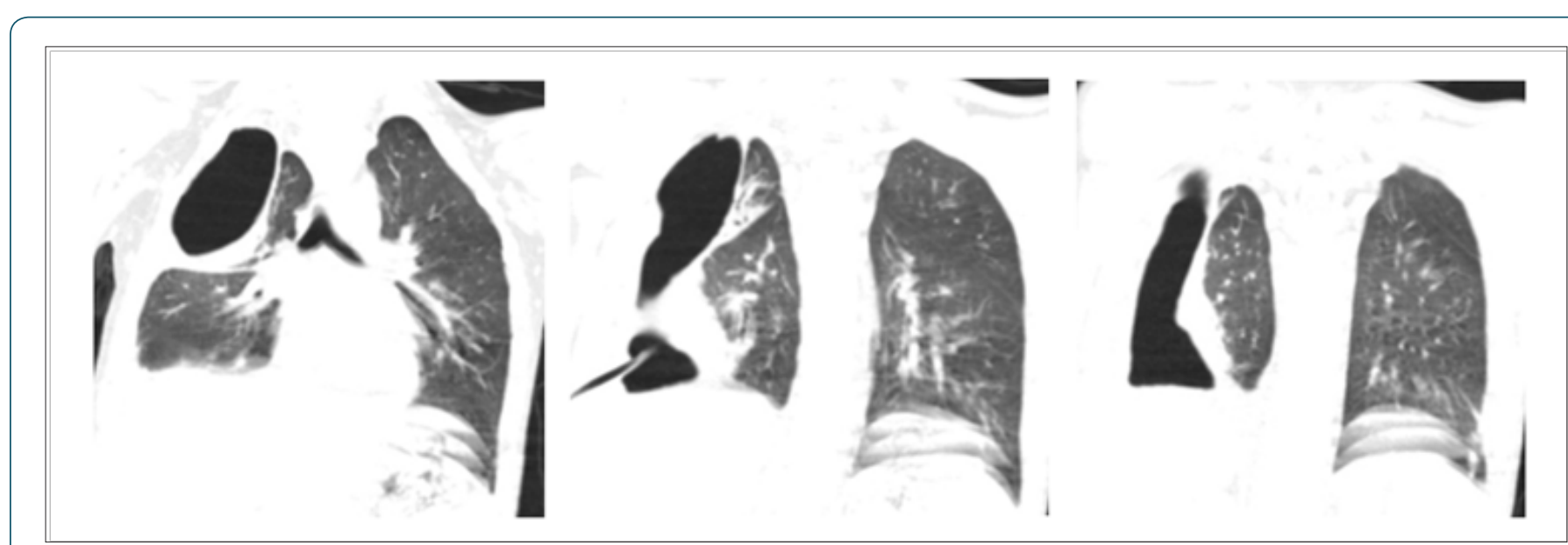

Figure 1: CT scan, direct projection. The presence of fluid and free gas in the pleural cavity, a shift of the mediastinum to the healthy side is noted.

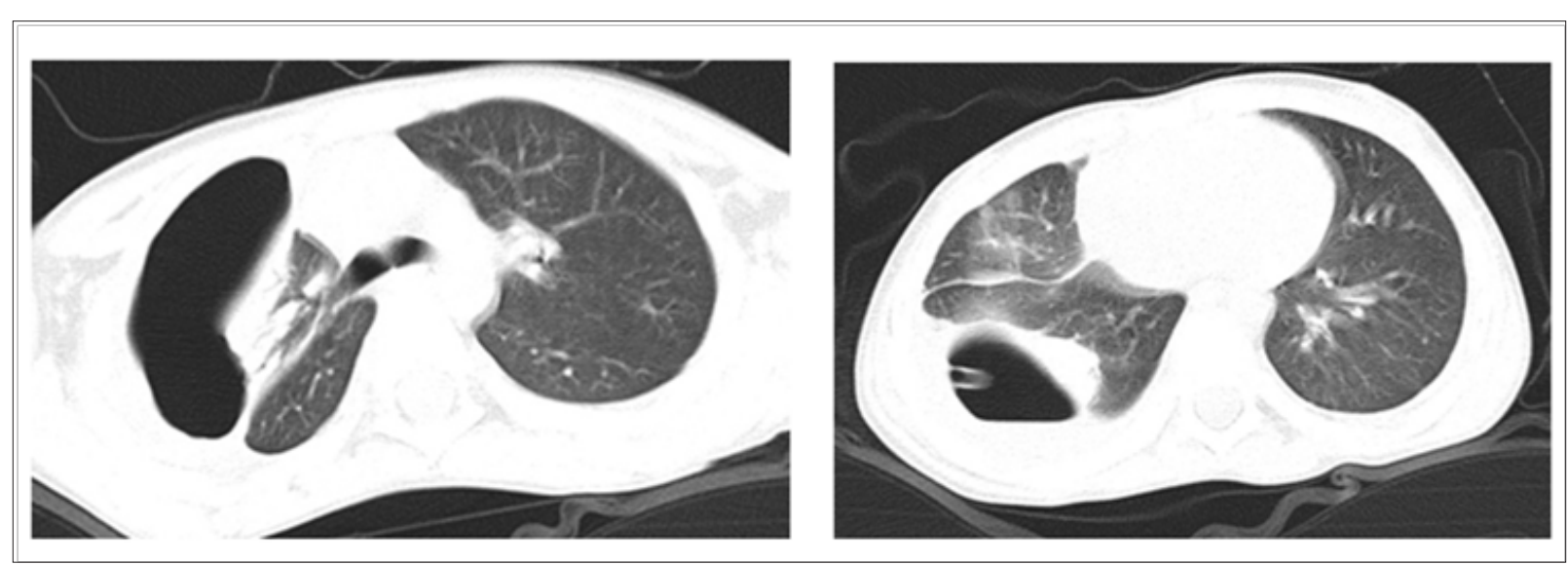

Figure 2: CT scan. The air is not connected with the lungs, the presence of a fistula connected with the bronchial tree.

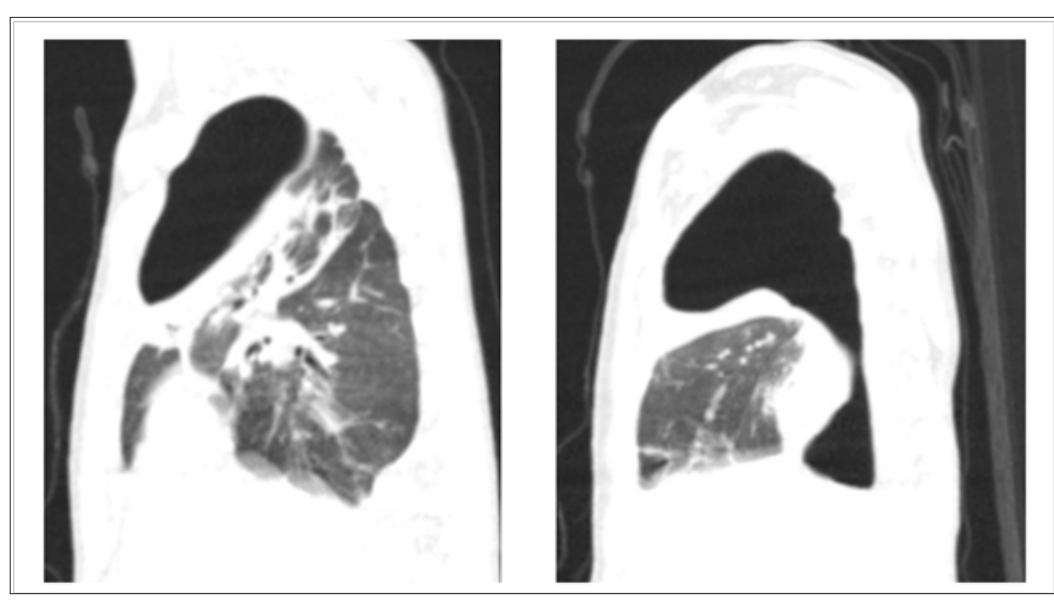

Figure 3: The lateral projection of CT scan. The relation of the fistula to the root of the lung. 


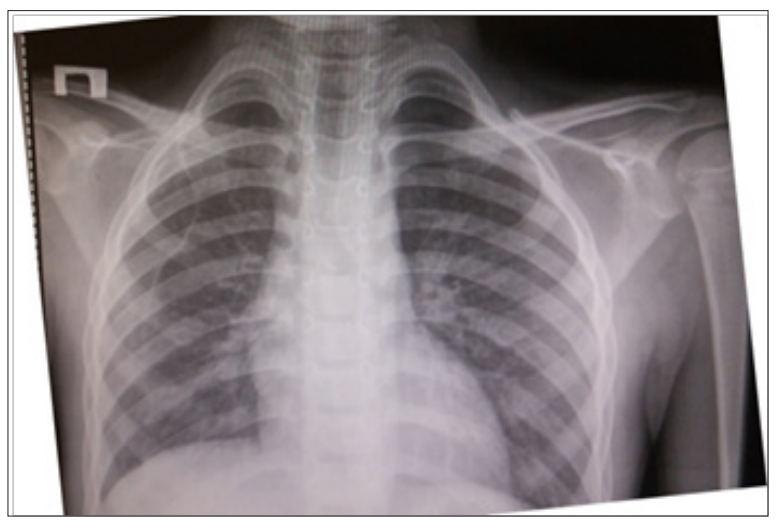

Figure 4: Chest X-ray in a direct projection. Complete expansion of the pleural cavity. Absence of fistula and free gas.

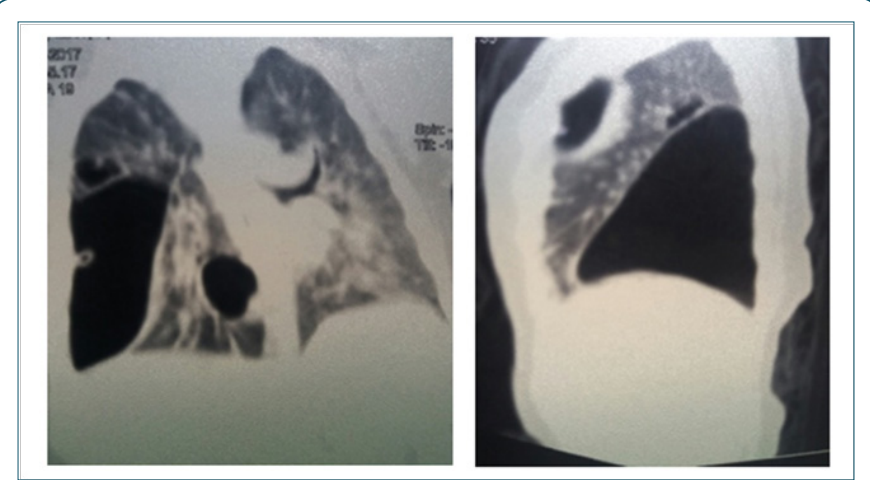

Figure 5: 3 moth of age patient's CT scan. Right-sided pneumothorax. Bronchopleural fistula.

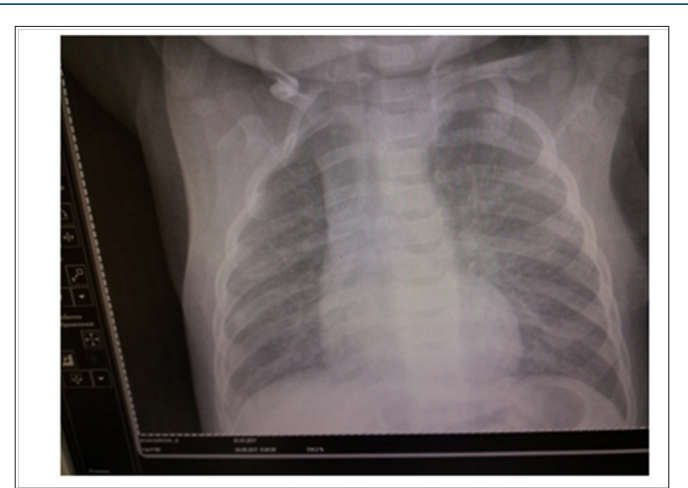

Figure 6: X-ray of chest after bronchial lavage and temporary bronchial occlusion.

\section{Conclusion}

After the clinical, radiological and bronchological evaluation of the effectiveness of temporary bronchial occlusion in the treatment of Bronchopleural fistula in children, we came to the conclusion:

a) Temporary bronchial occlusion promoted evacuation and obliteration of foci of destruction in the lung tissue in conditions of temporary atelectasis of the affected parts of the lung with a constant aspiration of the contents from the pleural cavity b) A persistent vacuum was created in the pleural cavity as a result of its dissociation with the occludor with the bronchial tree

c) Occlusion of the affected parts prevented bronchogenic dissemination of the purulent infection, delimiting the healthy parts of the lungs

d) After occlusion of the fistula-bearing sections of the bronchi, favorable conditions were created to close the Bronchopleural fistulas as a result of the formation of adhesions between the visceral and parietal pleura, the formation of a limited fibrothorax

e) The residual pleural cavity was eliminated due to expansion and increase in the volume of the healthy part of the lung, displacement of the mediastinum, reduction of intercostal spaces and lifting of the diaphragm

Thus, the temporary occlusion of the bronchi in bacterial destruction of the lungs, with extrapulmonary complications, such as pyopnemothorax, allowed complete recovery in all sick children. The use of a large porous occludor impregnated with povidone iodine and tantalum, with a diameter up to 3 times increasing the diameter of the fistula, gives a rapid occlusion of the fistula, the elimination of "air leakage" and the spreading of the bronchus. After removal of the occludor, capillary blood flow improved and the anatomical shunting of blood in the small circle of blood circulation decreased significantly. Bronchography at longterm (up to 12 months) was an informative method for diagnosing intrapulmonary cavities and showed a picture of complete recovery after occlusion.

\section{References}

1. Sheykhet YN, Tsceymakh EA (1996) Treatment of acute empyema. Barnaul, Russia.

2. Vagner EA, Kabanov AN (1993) Treatment of bronchial fistula. Medicine, Moscow, Russia.

3. Varoli F, Scalambra SM, Mariani C (1988) Endoscopic treatment of bronchopleural fistula: a personal method Lectur book of XXYI World Congress of the International College of Surgeons. Milan pp. 341-343.

4. Perepelicyn VN (1996) Minimally invasive methods of surgical treatment of non-specific empyema. Dissertation of Doctor of Science Perm, Russia p. 39.

5. McManigle JE, Fletcher GL, Tenholder MF (1990) Bronchoscopy in the management of bronchopleural fistula. Chest 97: 1235-1238.

6. Hollaus PH, Lax F, Janakiev D, Lucciarini P, Katz E, et al. (1998) Endoscopic treatment of postoperative bronchopleural fistula: experience with 45 cases. Ann Thorac Surg 66(3): 923-927.

7. Lois M, Noppen M (2005) Bronchopleural fistulas: an overview of the problem with special focus on endoscopic management. Chest 128(6): 3955-3965.

8. Rafinski R (1968) Die zeitweilige piombirung des als behandiungsmethode des spontanpneumothorax Mschr. Kinderhelok 116(8): 471-475.

9. Geraskin VI, Shtykhno OF, Kuleshov BV (1974) Treatment of bronchopleural fistula with temporary bronchial occlusion of collapsed lung. Surgery p. 36-42. 
10. Uchida T, Wada M, Sakamoto J, Arai Y (1999) Treatment for empyema with bronchopleural fistulas using endobronchial occlusion coils: report of a case. Surg Today 29(2): 186-189.

11. Turk AE, Karanas YL, Cannon W, Chang J (2000) Staged closure of complicated bronchopleural fistulas. Ann Plastic Surgery 45(5): 560564.
12. Spieden V (1993) Surgical treatment of thoracic empyema. Rozhl Chir 72(6): 275-278. (c) ()

This work is licensed under Creative Commons Attribution 4.0 License

Submission Link: Submit Article

DOI: $10.32474 /$ PAPN.2018.01.000105

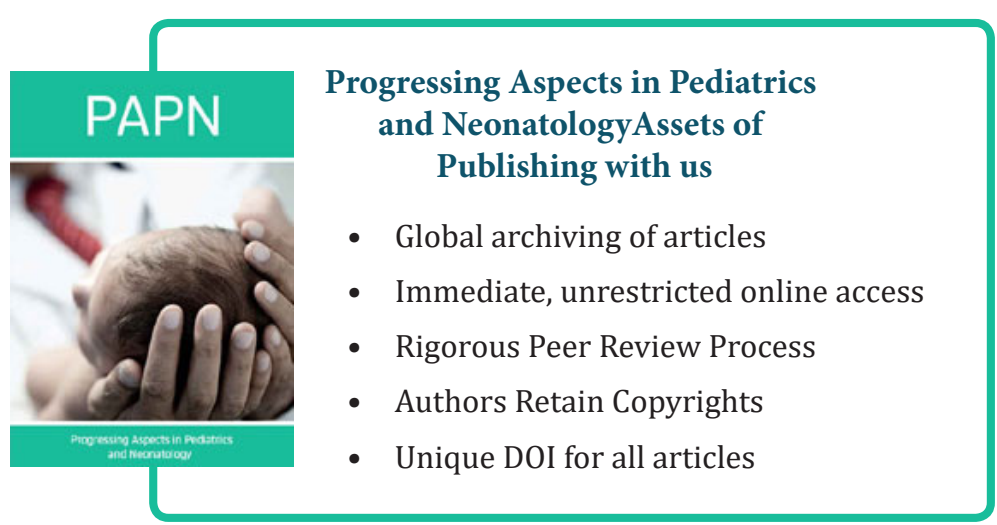

